\title{
Performance studies of resistive Micromegas detectors for the upgrade of the ATLAS Muon spectrometer
}

\author{
Fabian Kuger, for the ATLAS Collaboration \\ CERN, Geneva, Switzerland
}

\section{A R T I C L E I N F O}

\section{Article history:}

Received 25 March 2016

Received in revised form

4 April 2016

Accepted 1 June 2016

Available online 2 June 2016

Keywords:

Resistive Micromegas

ATLAS Muon Spectrometer

New Small Wheel

\begin{abstract}
A B S T R A C T
With the high luminosity upgrade of the LHC the ATLAS Muon spectrometer will face increased particle rates, requiring an upgrade of the innermost end-cap detectors with a high-rate capable technology. Micromegas have been chosen as main tracking technology for this New Small Wheel upgrade. In an intense R\&D and prototype phase the technology has proven to meet the stringent performance requirements of highly efficient particle detection with better than $100 \mu \mathrm{m}$ spatial resolution, independent of the track incidence angle up to $32^{\circ}$, in a magnetic field $\mathrm{B} \leq 0.3 \mathrm{~T}$ and at background hit rate of up to $15 \mathrm{kHz} / \mathrm{cm}^{2}$.

(c) 2016 The Author. Published by Elsevier B.V. This is an open access article under the CC BY license (http://creativecommons.org/licenses/by/4.0/).
\end{abstract}

\section{Introduction - Micromegas for the ATLAS NSW}

Resistive Micromegas (MICRO MEsh GAseous Structure) detectors have proven over the years to be a reliable high rate capable detector technology characterized by an excellent spatial resolution and discharge immunity. The ATLAS collaboration [1] has chosen the resistive Micromegas technology, along with smallstrip Thin Gap Chambers (sTGC), for the high luminosity upgrade of the inner muon station in the high-rapidity region, the so called New Small Wheel (NSW) upgrade project [2].

The NSW shall provide a muon $p_{T}$ measurement with $10 \%$ resolution at $1 \mathrm{TeV} / \mathrm{c}$. This requires fully efficient Micromegas chambers with spatial resolution better than $100 \mu \mathrm{m}$ independent of the track incidence angle $\left(\leq 32^{\circ}\right)$, the magnetic field $(B \leq 0.3 \mathrm{~T})$ and with a rate capability of up to $15 \mathrm{kHz} / \mathrm{cm}^{2}$.

The design of the Micromegas quadruplets foreseen for the NSW upgrade is introduced in Section 2. Different prototypes, starting with small $\left(10 \times 10 \mathrm{~cm}^{2}\right)$ single plane detectors, medium size $\left(0.95 \times 0.5 \mathrm{~m}^{2}\right)$ Micromegas quadruplets to full size NSW modules are presented in Section 3. An intense series of tests has been performed using cosmic muons as well as medium (10 GeV/c) and high (150 GeV/ c) momentum muon and hadron beams at CERN including measurements inside magnetic field and in high-rate gamma background environments. Results on the measured efficiency and position resolution will be shown in Section 4, demonstrating the excellent properties of the detectors meeting the NSW requirements.

\section{Design of the NSW Micromegas modules}

Micromegas detectors [3] are gaseous parallel plate detectors consisting of two gas filled volumes with asymmetric electric fields (Fig. 1): the drift region $(5 \mathrm{~mm}, 0.6 \mathrm{kV} / \mathrm{cm})$ and the amplification region $(128 \mu \mathrm{m}, 40-45 \mathrm{kV} / \mathrm{cm})$. The two regions are separated by a thin metallic mesh and are filled with an $\mathrm{ArCO}_{2}$ 93:7 vol\% gas mixture. In the detector layout adopted for the ATLAS NSW upgrade a layer of resistive strips covers the readout strips, making the detector discharge tolerant [4]. Grounding the mesh and applying positive high voltage to the resistive anode strips allows a spatial segmentation of the amplification voltage, according to the segmentation of the resistive layer.

A quadruplet design has been chosen for the NSW Micromegas to accommodate four detector layers within the $8 \mathrm{~cm}$ wide envelope of a module (Fig. 2). The four readout layers are paired in a back-to-back configuration on two double-sided readout panels. The woven stainless steel mesh is mechanically pressed onto $128 \mu \mathrm{m}$ high pillars distributed with $7 \mathrm{~mm}$ distance over the full anode surface and electro-statically pulled into position once voltages are applied. The drift panels, carrying the cathode, the gas distribution pipes, the gas gap defining frames and the metallic mesh, are arranged in a central double-sided panel and two singlesided panel on the outside (Figs. 3 and 4).

To reach a spatial resolution of $100 \mu \mathrm{m}$, the $300 \mu \mathrm{m}$ wide readout strips are orientated perpendicularly to the precision coordinate on the two sides of the first panel (eta). The inclination of the strips by $\pm 1.5^{\circ}$ on the two sides of the second panel (stereo) allows for a coarse determination of the second coordinate, with an accuracy of $2-3 \mathrm{~mm}$ without significant degradation of the 
precision coordinate resolution [5].

The two NSWs will accommodate 128 Micromegas quadruplets of 4 different type and size. Each type will be fabricated in a different construction center and the NSW assembly will be done at CERN.

\section{Prototypes and mechanical accuracy requirements}

In preparation of the NSW construction several prototypes were built to demonstrate that performance requirements can be met and construction methods are mature to reach the required mechanical accuracy. Among these mechanical constraints the two most challenging are the surface planarity requirement of $\pm 80 \mu \mathrm{m}$ (RMS) over several square meters surface and the strip position accuracy of better than $30 \mu \mathrm{m}$ between the four planes of one quadruplet detector.

\subsection{Small size, single-plane Micromegas}

The hit reconstruction performance of small $\left(10 \times 10 \mathrm{~cm}^{2}\right)$ single-plane Micromegas was tested with muons and hadrons provided by the CERN PS or SPS testbeam facilities. The chambers were arranged in a telescope configuration and were rotated within a frame to study tracks crossing under different inclination angles.

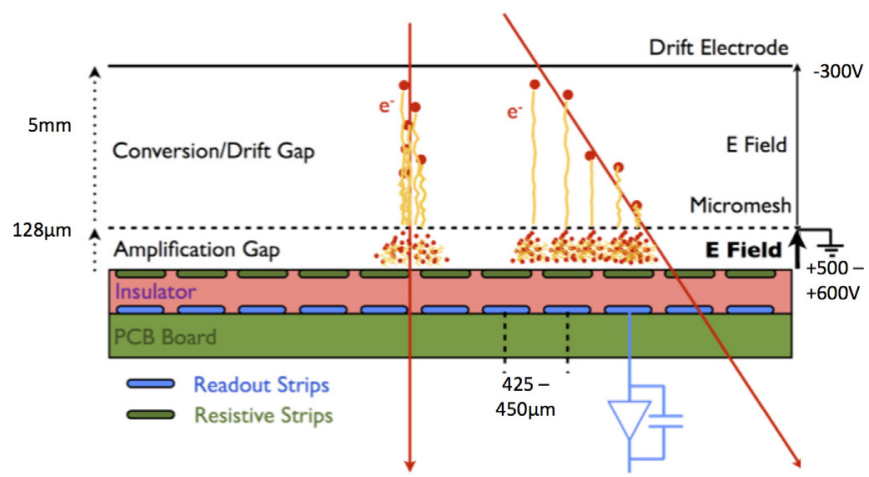

Fig. 1. Schematic of a resistive Micromegas with the dimensions and voltages foreseen for the NSW detectors. A signal formation process is shown for a perpendicular and an inclined track. Not to scale.

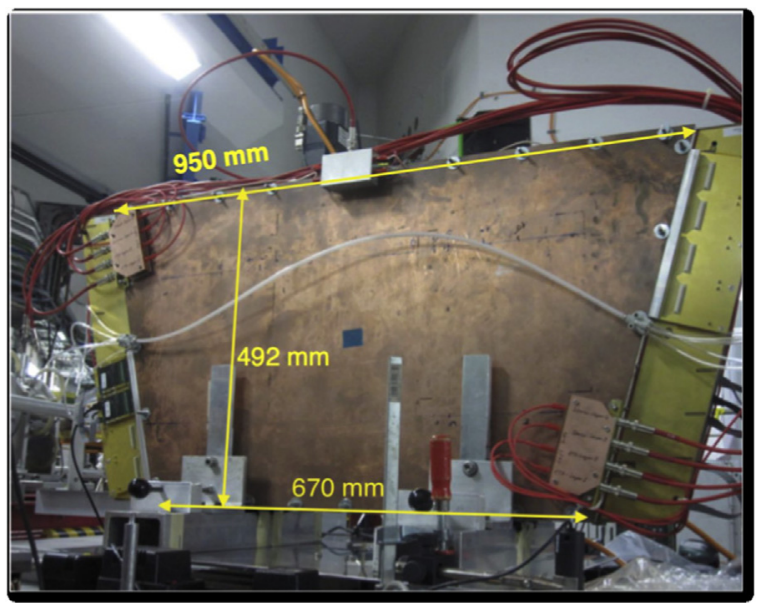

Fig. 3. The MMSW Quadruplet detector equipped with HV cables, gas pipes and partially with APV25 electronics. [6].

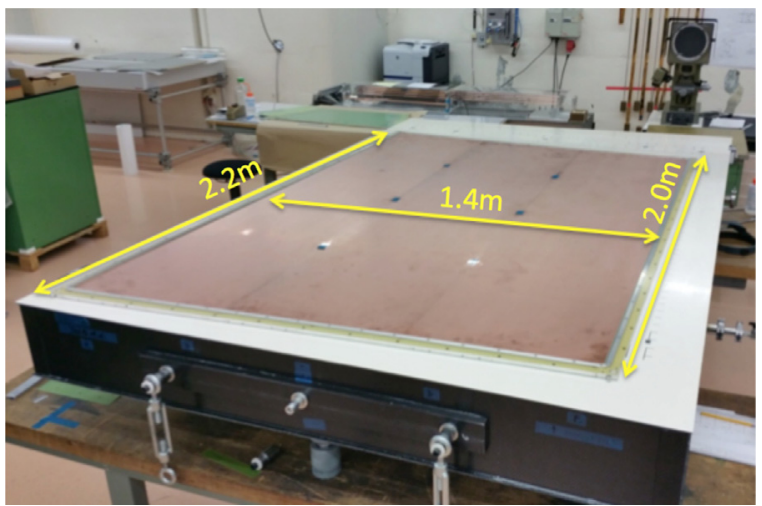

Fig. 4. Full size drift panel of a LM2 Micromegas Module build at CERN.

\subsection{Medium size quadruplet prototypes}

Two Micromegas Small Wheel (MMSW) prototype quadruplets were built, following the layout of the NSW detectors described in Section 2, despite their reduced size $\left(0.95 \times 0.5 \mathrm{~m}^{2}\right)$ compared to the full NSW modules.

For the MMSW detectors a panel planarity of $50 \mu \mathrm{m}$ (RMS) has been achieved. The plane-to-plane alignment of the two sides of a panel has been measured to be better than $30 \mu \mathrm{m}$ in the precision coordinate direction. A detailed report on the construction,

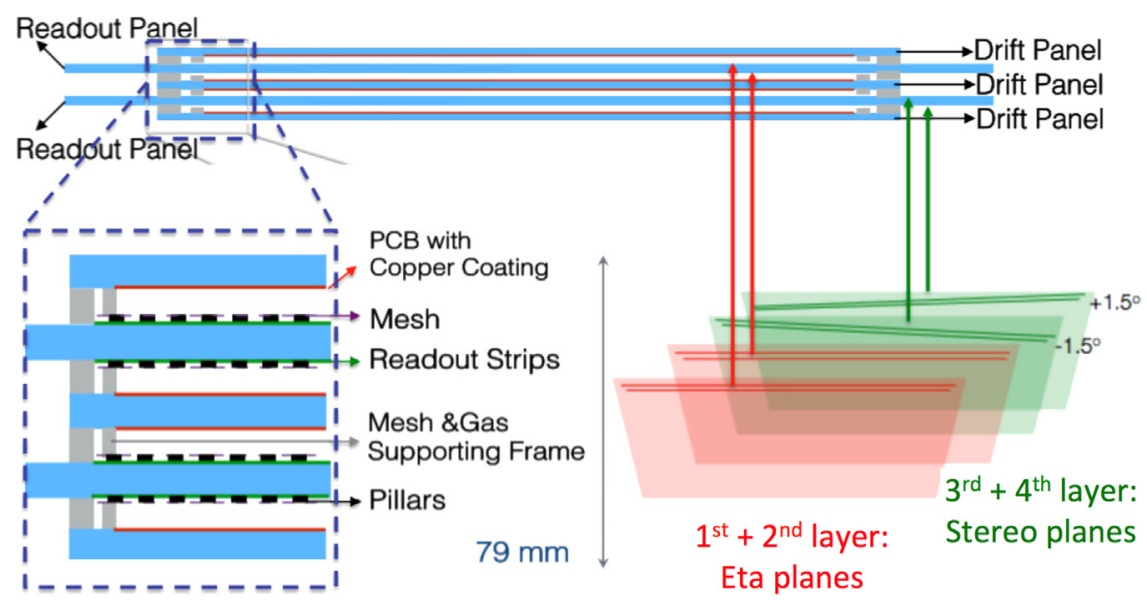

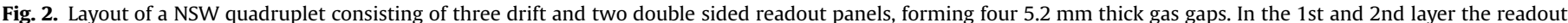
strips are parallel to each other and perpendicular to the radial direction (eta), in the 3rd and 4 th layer the strips are inclined by $\pm 1.5^{\circ}$ (stereo). 
validation and performance is presented in [6].

\subsection{NSW size modules}

First Micromegas modules in full NSW size are currently under construction and several panels have already been completed in all construction sites. Profiting from revised and improved construction methods, compared to the MMSW prototype, a planarity better than $30 \mu \mathrm{m}$ (RMS) on $2-3 \mathrm{~m}^{2}$ surface has been achieved.

\section{Hit reconstruction performance}

\subsection{Spatial resolution in the precision coordinate}

The spatial resolution of the Micromegas detectors was investigated during a series of tests using medium $(10 \mathrm{GeV} / \mathrm{c})$ and high $(150 \mathrm{GeV} / \mathrm{c})$ momentum muon and hadron beams at CERN. A telescope setup of Micromegas detectors was used to determine the tracks and the residuals between the hit position in two Micromegas chambers were measured and divided by $\sqrt{2}$ to obtain the single-plane resolution. For perpendicularly incident beam the hit position was defined by weighting the strip positions with the charge measured on each strip (centroid method). The residual distributions were fitted with the sum of two Gaussians. The spatial resolution of a single plane corresponds to the weighted average of the two fitted Gaussians (Fig. 5):

$\sigma_{\text {weight }}^{2}=f_{\text {core }} \cdot \sigma_{\text {core }}^{2}+f_{\text {tails }} \cdot \sigma_{\text {tails }}^{2}$,

with their corresponding weighting factors $f_{\text {core,tails }}$.

The requirement of a spatial resolution better than $100 \mu \mathrm{m}$ in the precision coordinate was met with various small-size Micromegas and with each of the MMSW quadruplet eta-planes and the combined stereo-planes.

\subsection{Centroid vs. $\mu$ TPC reconstruction method}

The NSW will be hit by muons under an angle of up to $32^{\circ}$. The centroid method is accurate for hit position reconstruction of almost perpendicular tracks, but the resolution deteriorates with increasing track incident angle, since the signal extends over a larger number of strips. By measuring the arrival time of ionization

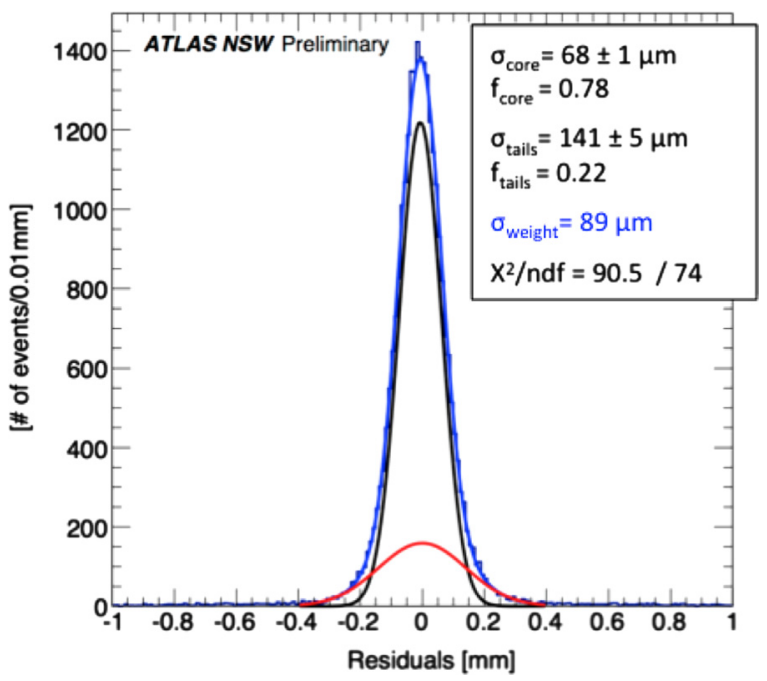

Fig. 5. Distribution of the residuals between the hit position in two T-type Micromegas chambers, divided by $\sqrt{2}$. The measurements were performed with an amplification voltage $\mathrm{HV}_{\mathrm{amp}}=550 \mathrm{~V}$. The data were acquired with a $150 \mathrm{GeV} / \mathrm{c} \mu / \pi^{+}$ beam.

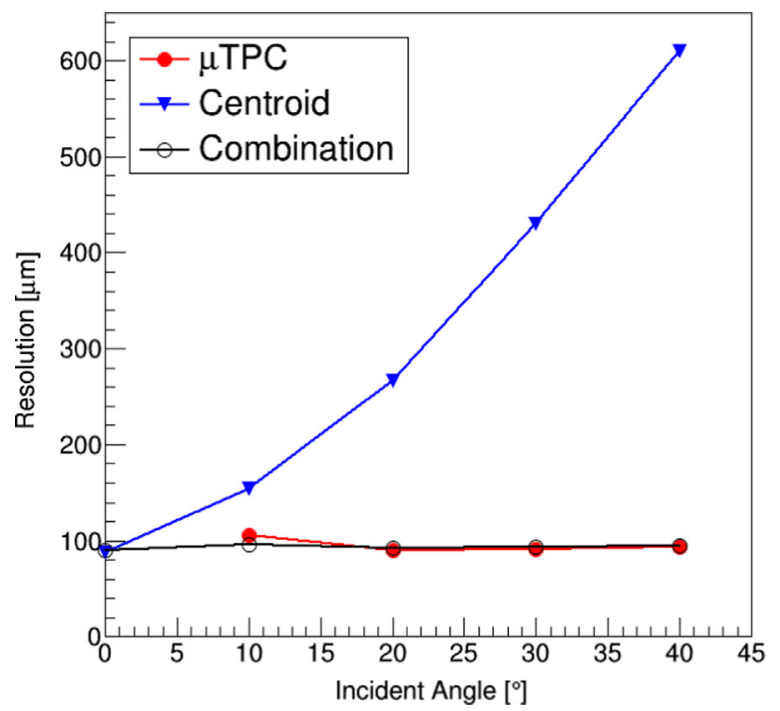

Fig. 6. Spatial resolution using the charge centroid method (blue triangles), the $\mu \mathrm{TPC}$ method (full red circles) and the combination of the two (black open circles) as a function of the particle incident angle. Lines are for optical guidance only [7].

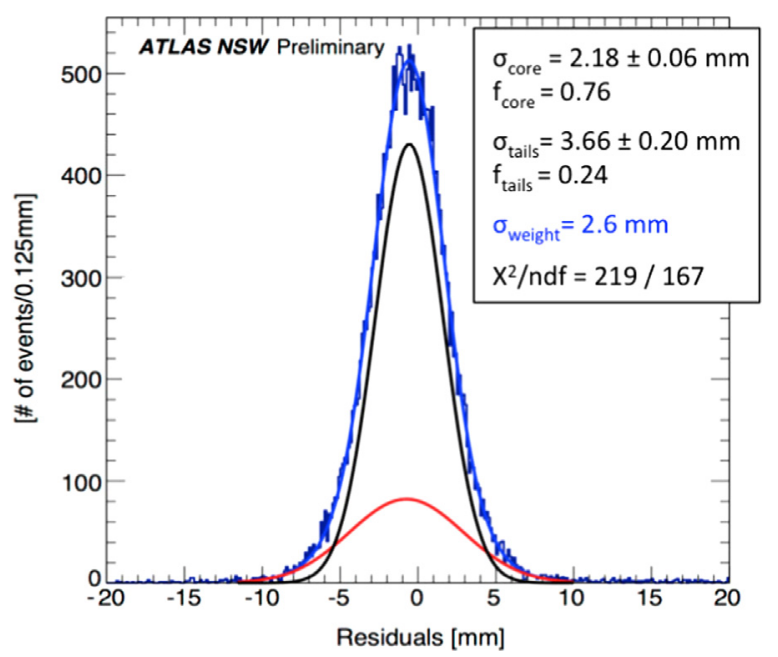

Fig. 7. Residual distributions from the hit position difference between the second coordinate hit reconstructed in the stereo layers of MMSW and the hit position extrapolated from the more accurate reference telescope. The measurements were performed with an amplification voltage $H V_{a m p}=580 \mathrm{~V}$. The data were acquired with a $6 \mathrm{GeV} / \mathrm{c} \mu / \pi^{+}$beam.

charge on the strips, the origin of the primary ionization in the drift gap can be determined and the track can be reconstructed in a single detector plane ( $\mu \mathrm{mPC}$ method). The hit position is defined as the track position in the middle of the drift gap.

With a $5 \mathrm{~mm}$ drift gap and $\mathrm{O}(400 \mu \mathrm{m})$ strip pitch the $\mu \mathrm{TPC}$ method can be used for incident angles $\geq 10^{\circ}$. A combination of both methods provides the required $100 \mu \mathrm{m}$ spatial resolution over the full incident angle range (Fig. 6).

\subsection{Spatial resolution in the second coordinate}

Combining the hit position measurement on the two stereo layers with strip orientation rotated by $\pm 1.5^{\circ}$, the hit coordinate can be reconstructed in the precision and the second coordinate [5].

The resolution of the MMSW second coordinate stereo doublet hit reconstruction was measured in a $\mu / \pi^{+}$beam, with one reference chamber for the independent measurement of the second 


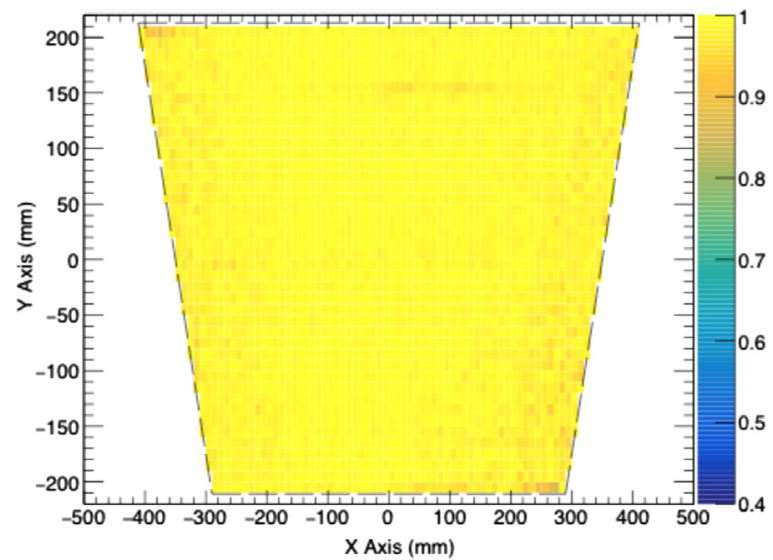

Fig. 8. Reconstruction efficiency of the 1st MMSW layer over the detector surface with an amplification voltage of $580 \mathrm{~V}$. Track positions of cosmic Muons were determined with the 2 nd eta layer and the combined $3 \mathrm{rd}+4$ th layer stereo information.

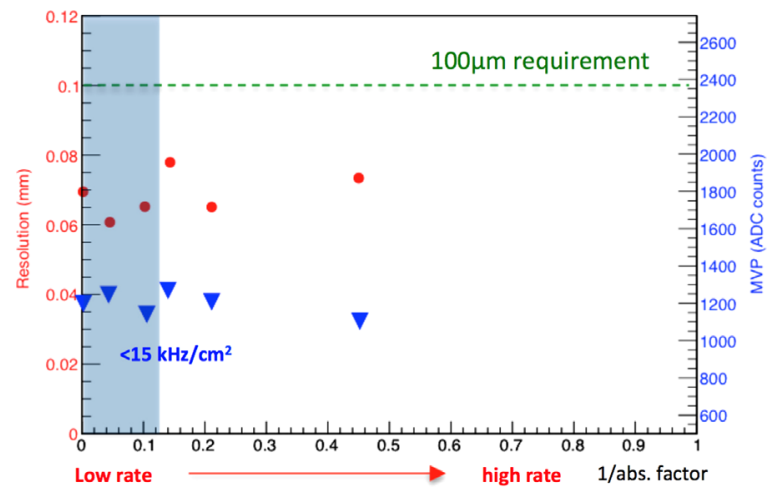

Fig. 9. Spatial resolution (red circles) and most propable pulse height for muonsignals (blue triangles) over background intensity of $\gamma$-irradiation from a ${ }^{137} \mathrm{CS}$ source in GIF+ + in units of $1 /$ nominal absorption factor. The $<15 \mathrm{kHz} / \mathrm{cm}^{2}$ region (shaded) corresponds to maximum expected NSW background in high luminosity LHC operation.

coordinate. Both chambers were orientated perpendicularly to the beam axis and the hit position per layer was reconstructed using the centroid method.

The observed spatial resolution of $\sigma_{\text {stereo }}=2.6 \mathrm{~mm}$ is in agreement with the predicted ratio of $\sigma_{\text {stereo }} / \sigma_{\text {eta }}=27$ [5] and meets the ATLAS NSW requirement (Fig. 7).

\subsection{Detection efficiency}

A single-plane particle detection efficiency of $98 \%$ is required over the full surface of the NSW Micromegas detectors. The efficiency of each MMSW detector plane (Fig. 8) was measured by reconstructing cosmic muon tracks in 3 out of 4 layers and requiring a corresponding hit in the tested layer [6].

\subsection{Performance in high-rate background environment}

The NSW detectors closest to the beam pipe will be exposed to a background hit rate of up to $15 \mathrm{kHz} / \mathrm{cm}^{2}$ after the high luminosity upgrade of the LHC [2]. The high-rate capability of the Micromegas was tested under $\gamma$-irradiation of a $16 \mathrm{TBq}{ }^{137} \mathrm{Cs}$ source in the CERN GIF ++ facility. A telescope was used to measure the tracks of high-momentum muons provided by the SPS secondary beam line and determine the resolution of the chamber as described in Section 4.1 [8].

No deterioration of the spatial resolution or the charge per signal was observed for hit rates up to $64 \mathrm{kHz} / \mathrm{cm}^{2}$ (Fig. 9).

\subsection{Performance in magnetic fields}

In the presence of a magnetic field perpendicular to the electric field the electrons are deflected from the electric field lines direction by the Lorentz angle. This deflection causes a systematic displacement of the reconstructed hit position compared to the zero magnetic field case (Fig. 10 -left). A correction of this shift can be applied during the reconstruction if the magnetic field strength and direction is known. The correlation between the position shift, the Lorentz angle and the magnetic field was studied in a beam test at CERN SPS $\mathrm{H} 2$ with the Micromegas setup in $\leq 1 \mathrm{~T}$ magnetic field [9].

After proper calibrated reconstruction, no degradation of the performance of the Micromegas detectors was observed and measurements of the Lorentz angle are in agreement with simulation prediction for the various magnetic fields (Fig. 10 - right).
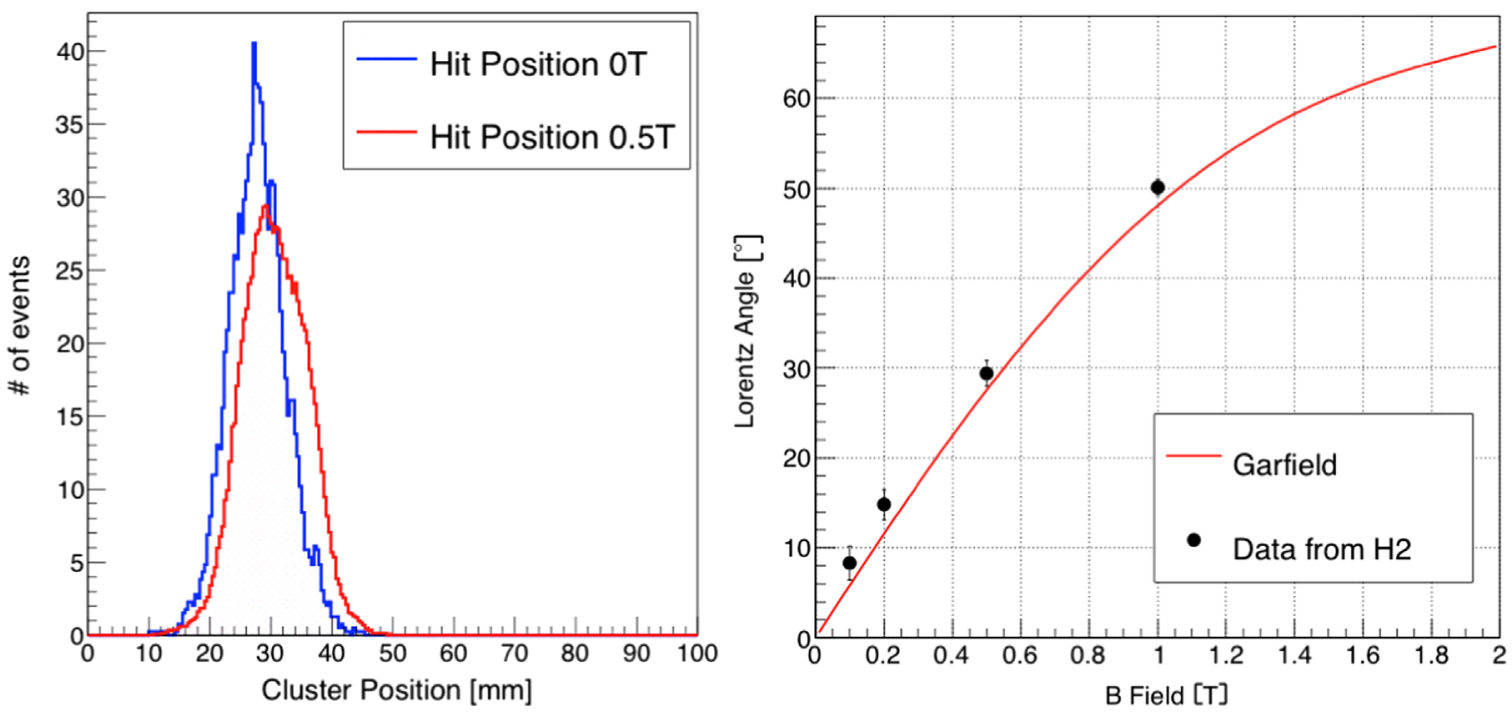

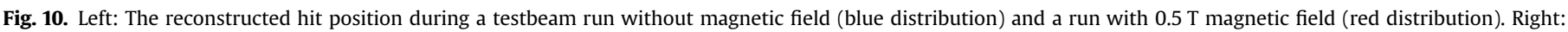

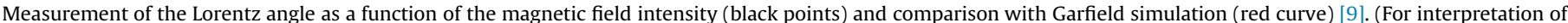
the references to color in this figure legend, the reader is referred to the web version of this article.) 


\section{Conclusion}

The challenging requirements on the performance of the NSW tracking detectors have proven to be achievable with the resistive Micromegas technology. An intense prototype- and R\&D-phase has confirmed the excellent hit reconstruction capabilities of these detectors, even under the difficult conditions of magnetic field and high background irradiation expected in the forward region of the ATLAS Muon spectrometer during high luminosity LHC operation. These performance results have been confirmed with quadruplet prototypes following the layout of the NSW modules on a reduced size. The first full size modules are currently under construction and so far all mechanical requirements have been met. Their finalization is expected in summer 2016 and will be followed by a re-validation of the detector performance on full scale.

\section{References}

[1] ATLAS Collaboration, The ATLAS Experiment at the CERN Large Hadron Collider, JINST 3 (2008) S08003.

[2] ATLAS Collaboration: ATLAS New Small Wheel Technical Design Report, CERNLHCC-2013-006, ATLAS-TDR-020-2013, Geneva. 〈https://cds.cern.ch/record/ 1552862>, 2013.
[3] Y. Giomataris, et al., MICROMEGAS: a high-granularity position-sensitive gaseous detector for high particle-flux environments, Nucl. Instrum. Methods A 376 (1996) 29-35.

[4] T. Alexopoulos, et al., A spark-resistant bulk-micromegas chamber for high-rate applications, Nucl. Instrum. Methods A 640 (2011) 110-118.

[5] T. Alexopoulos, et al., Stereo information in micromegas detectors, ATL-MUONPUB-2015-001, CERN, Geneva, 2015.

[6] M. Bianco, et al., Construction of two large-size four-plane micromegas detectors, Nucl. Instrum. Methods A 814 (2016) 117-130.

[7] M. Iodice, et al., Performance studies of MicroMegas for the ATLAS experiment JINST 9 (2014) C01017.

[8] B. Alvarez Gonzalez, et al., Ageing Studies on the First Resistive-MicroMeGaS Quadruplet at GIF++, ATL-MUON-PROC-2015-021, CERN, Geneva, 2015.

[9] K. Ntekas, et al., Micromegas Chambers for the ATLAS Muon Spectrometer Upgrade, ATL-MUON-PROC-2014-011, CERN, Geneva, 2014. 